\title{
Recorded time periods of bispectral index values equal to zero predict neurological outcome after out-of-hospital cardiac arrest
}

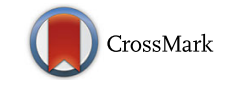

\author{
Ward Eertmans ${ }^{1,2^{*}}$ (D) Cornelia Genbrugge ${ }^{1,2}$, Gilles Haesevoets ${ }^{1,2}$, Jo Dens ${ }^{1,3}$, Willem Boer ${ }^{2}$, Frank Jans ${ }^{1,2}$ \\ and Cathy De Deyne ${ }^{1,2}$
}

\begin{abstract}
Background: Prognostication in out-of-hospital cardiac arrest (OHCA) survivors is often difficult. Recent studies have shown the predictive ability of bispectral index (BIS) monitoring to assist with early neuroprognostication. The aim of this study was to investigate whether characteristics of BIS values equal to zero (BIS 0) (i.e. duration and/or uni- versus bilateral presence) instead of simply their occurrence are better indicators for poor neurological outcome after OHCA by aiming at a specificity of $100 \%$.
\end{abstract}

Methods: Between 2011 and 2015, all successfully resuscitated OHCA patients were treated with targeted temperature management (TTM) at $33^{\circ} \mathrm{C}$ for 24 hours followed by rewarming over 12 hours $\left(0.3^{\circ} \mathrm{C} / \mathrm{h}\right)$. In total, BIS values were registered in $77 \mathrm{OHCA}$ patients. The occurrence of unilateral (BIS 0 at one hemisphere) and bilateral (BIS 0 at both hemispheres) BIS 0 values as well as their total duration were calculated. Receiver operating characteristic (ROC) curves were constructed using the total duration with BIS 0 values calculated from the initiation of TTM onwards to determine poor neurological outcome.

Results: In 30 of 77 OHCA patients (39\%), at least one BIS 0 value occurred during the first 48 hours after admission. Of these 30 patients, six (20\%) had a good (cerebral performance category (CPC) 1-2) and 24 (80\%) a poor neurological outcome (CPC3-5) at 180 days post-CA. Within these 30 patients, the incidence of bilateral BIS 0 values was higher in patients with poor neurological outcome (CPC1-2: 2 (33\%) vs. CPC3-5: 19 (79\%); $p=0.028)$. The presence of a BIS 0 value predicted poor neurological outcome with a sensitivity of $62 \%$ and specificity of $84 \%$ (AUC: $0.729 ; p=0.001$ ). With a ROC analysis, a total duration of 30,3 minutes with BIS 0 values calculated over the first 48 hours predicted poor neurological outcome with a sensitivity of $63 \%$ and specificity of $100 \%$ (AUC: $0.861 ; p=0.007$ ).

Conclusions: This study shows that a prolonged duration with (bilateral) BIS 0 values serves as a better outcome predictor after OHCA as compared to a single observation.

Keywords: Cardiac arrest, Prognostication, Neuromonitoring, Targeted temperature management

\section{Background}

Despite improvements in cardiopulmonary resuscitation and intensive care treatment including targeted temperature management (TTM), hypoxic-ischaemic brain injury remains the predominant cause of death in out-of-hospital cardiac arrest (OHCA) patients admitted

\footnotetext{
* Correspondence: ward.eertmans@uhasselt.be

${ }^{1}$ Department of Medicine and Life Sciences, Hasselt University, Diepenbeek, Belgium

${ }^{2}$ Department of Anaesthesiology, Intensive Care, Emergency Medicine and Pain Therapy, Ziekenhuis Oost-Limburg, Genk, Belgium

Full list of author information is available at the end of the article
}

to the intensive care unit [1-3]. Early and reliable identification of patients with no prospect of favourable outcome avoids futile and expensive treatment prolongation and would be of value informing relatives. Currently, a multimodal strategy is being recommended for reliable neuroprognostication, encompassing modalities such as electro-encephalography (EEG), somatosensory evoked potentials (SSEP) and magnetic resonance imaging [4-7]. Still, most of these robust outcome predictors are labour-intensive, expensive, not continuous and above all require trained specialists for correct 
interpretation. Recently, the prognostic performance of simple bispectral index (BIS) monitoring has been investigated thoroughly in the post-cardiac arrest (CA) setting [8-15]. This monitoring option, originally designed to monitor the degree of awareness during anaesthesia, converts raw sampled frontal EEG signals into a simple and real-time BIS index that ranges from 0 (iso-electric EEG) to 100 (normal electrical activity in awake subjects). The presence of BIS values equal to zero (BIS 0) during TTM at $33{ }^{\circ} \mathrm{C}$, equivalent to flat or low-voltage EEG, has been associated with poor neurological outcome [8]. However, as it has been shown that the presence of BIS 0 values does not reach a specificity of $100 \%$ on its own, it was recommended not to use the presence of low-voltage EEG or BIS 0 values on its own to predict poor outcome after OHCA [16]. We investigated whether characteristics of BIS 0 values (duration and/or uni- versus bilateral presence) during the first 48 hours after admission instead of simply their occurrence were indicative for poor neurological outcome after OHCA aiming at a specificity of $100 \%$.

\section{Methods}

In this prospective, observational study, all adult comatose survivors after OHCA with a presumed cardiac origin admitted to the coronary care unit (CCU) of Ziekenhuis Oost-Limburg (Genk, Belgium) were consecutively included between March 2011 and May 2015. Ethical approval was obtained before study onset (CME11/066) and written informed consent was obtained from the patient's next of kin.

The institutional post-resuscitation protocol has been described previously $[17,18]$. In summary, TTM at $33{ }^{\circ} \mathrm{C}$ was initiated immediately after admission to the emergency department by administering cold fluids intravenously $\left(4{ }^{\circ} \mathrm{C}, 30 \mathrm{ml} / \mathrm{kg}\right)$. After admission to the CCU, TTM at $33{ }^{\circ} \mathrm{C}$ was further induced and maintained for 24 hours using either a surface${\text { cooling } \text { (ArcticGel }^{\mathrm{mt}} \text { pads, Artic Sun System }}^{\bullet}$ 5000, Medivance, Louisville, CO, USA) or endovascular cooling system (Icy-cathether, CoolGard ${ }^{\circledR} 3000$; Alsius, Irvine, CA, USA). Both systems were equipped with a feedback loop system to control target temperature using an oesophageal temperature probe. Twenty-four hours after CCU admission, patients were actively rewarmed towards a core temperature of $36.6{ }^{\circ} \mathrm{C}$ at a rate of $0.3{ }^{\circ} \mathrm{C}$ per hour. All patients were intubated, mechanically ventilated and sedation was induced and maintained by administering propofol, midazolam and remifentanil intravenously. Within the period of TTM, doses of sedative drugs were titrated to obtain values between -3 and -5 on the Richmond Agitation-Sedation Scale. According to the guidelines, cisatracurium was only administered in case of shivering [19]. EEGs were carried out on clinical indication and epileptic activity was treated with anti-epileptic drugs. Patients were extubated once their neurological, hemodynamic and respiratory status was recovered sufficiently.

In patients remaining comatose despite complete cessation of sedation, full supportive treatment was continued until at least 72 hours after rewarming. As such, withdrawal of life-sustaining therapy was never performed before this time point. In accordance with recommendations of international guidelines, signs of brain death (i.e. absent pupillary and corneal reflexes), refractory seizures and the bilateral absence of the N20 component of the SSEPs were taken into account for the decision to withdraw life support [20].

BIS VISTA ${ }^{\mathrm{mm}}$ monitoring with a six-electrode frontotemporal bilateral sensor was initiated after CCU admission (Aspect Medical Systems, Inc. Norwood, MA, USA). BIS values were stored per second during the first 48 hours, resulting in a maximum of 172,800 unilateral data points per patient. In addition, the signal quality index (SQI) and electromyographic (EMG) power were recorded continuously. The SQI refers to the signal accuracy where values above $80 \%$ are considered as reliable. The EMG power, (measured as $\mathrm{dB}$ ), describes the electromyographic content of the EEG signal. The BIS VISTA $^{\text {mix }}$ device displays EMG bars according to the level of EMG noise where absence of EMG bars is indicative for EMG noise below $30 \mathrm{~dB}$ (i.e. a good signal quality) and presence of EMG bars means increasing EMG noise (1 bar: $30-38 \mathrm{~dB}$ up to 4 bars: $>55 \mathrm{~dB}$ ). Although physicians were not blinded to values displayed on the BIS monitor, decisions to withdraw life support or limit care were never based on the observed BIS values.

Outcome was assessed at 180 days post-CA using the Cerebral Performance Category (CPC) scale [21]. According to the scale classification, $\mathrm{CPC} 1$ is indicative for good cerebral performance; CPC2 implies a moderate disability with sufficient cerebral functioning for independent daily-life activity; СРC3 indicates severe neurological sequelae; CPC4 implies coma or vegetative state and $\mathrm{CPC} 5$ stands for death. In this study, a $\mathrm{CPC} 1-2$ and $\mathrm{CPC} 3-5$ was considered as good and poor neurological outcome, respectively.

Statistical analysis was performed using SPSS Version 24.0 (IBM Corp., Armonk, NY, USA). Equal distribution was tested by means of a Kolmogorov-Smirnov test. Depending on normality, categorical data were compared between patients with a good and poor neurological outcome using a Fisher exact or Chi-square test while unpaired $t$ tests or Mann-Whitney $U$ tests were used to compare continuous data. All data are presented as median (interquartile range (IQR)). Raw sampled BIS 
data were synchronized with the time TTM at $33{ }^{\circ} \mathrm{C}$ was initiated. The occurrence of unilateral (BIS 0 at left or right hemisphere) and bilateral (BIS 0 at both hemispheres) BIS 0 values was calculated together with their total duration within the first 48 hours from the initiation of TTM at $33{ }^{\circ} \mathrm{C}$ onwards. The concomitant EMG power and SQI values at the time BIS 0 values were present were analysed as well. The total duration with any BIS 0 value was calculated within the first 12 and 48 hours after TTM was initiated. Afterwards, receiver operating characteristic (ROC) curves were constructed using the total duration with any BIS 0 value (within the first 12 or 48 hours) to determine poor neurological outcome aiming at a specificity of $100 \%$. $P$ values $<0.05$ were considered as significant.

\section{Results}

One hundred and twenty-one eligible OHCA patients were prospectively enrolled between March 2011 and May 2015. Data of 44 patients were excluded from further analysis due to the following reasons: no registration of BIS values $(n=34)$, start BIS monitoring after day $2(\mathrm{n}=4)$ and incoherence between time notation and start BIS measurement $(n=6)$. In total, 77 successfully resuscitated OHCA survivors with a cardiac cause of origin were prospectively included (Fig. 1).

Forty-seven out of these 77 patients (61\%) never experienced a BIS value of 0 during the first 48 hours after CCU admission of whom 15 had a poor (32\%) and 32 (68\%) a good neurological outcome at 180 days post-CA. In contrast, at least one BIS 0 value was observed during the first 48 hours after CCU admission in 30 out of 77 patients (39\%). At the moment BIS 0 values were recorded, mean SQI was $98 \pm 1 \%$ and mean EMG was $26 \pm 3 \mathrm{~dB}$, indicating an adequate signal quality with insignificant interference. Of these 30 patients, six (20\%) had a good and 24 (80\%) a poor neurological outcome at 180 days post-CA. Baseline characteristics of these 30 patients are summarized in Table 1. Also, the incidence of bilateral BIS 0 values was higher in patients with a poor neurological outcome (CPC1-2: 2/6 (33\%) vs. CPC3-5: $19 / 24(79 \%) ; p=0.028)$. The presence of a BIS 0 value within the first 48 hours predicted poor neurological outcome with a sensitivity of $62 \%$ (95\% CI: 45-76) and specificity of $84 \%$ (95\% CI: 68-93) (AUC: 0.729 (0.614-0.844); $p=0.001 ;$ Fig. 2). This corresponded to a positive predictive value (PPV) of $80 \%$ (95\% CI: 61-92), a negative predictive value (NPV) of $68 \%$ (95\% CI: $53-80)$ and false positive ratio (FPR) of $20 \%$ (95\% CI: 8-39).

The median recording time in patients with a good and poor neurological outcome was 2880 (IQR: 27282880) and 2880 (IQR: 2521-2880) minutes, respectively $(p=0.631)$. The median duration with unilateral BIS 0

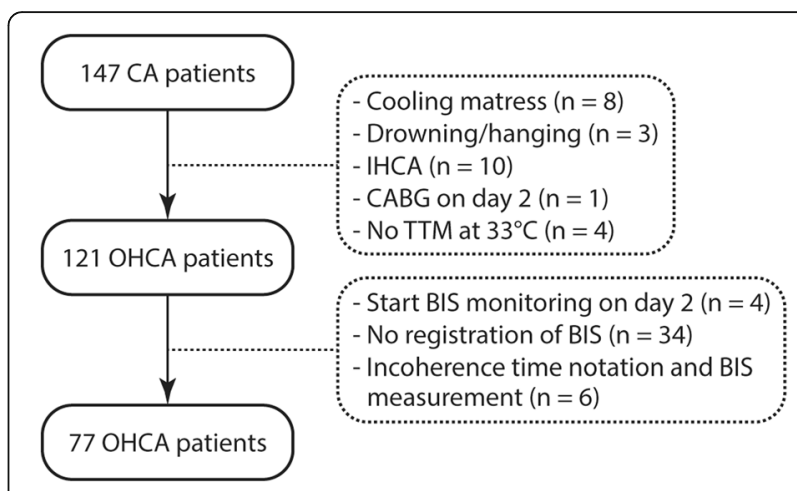

Fig. 1 Flowchart of enrolled study patients. CABG coronary-artery bypass graft surgery, IHCA in-hospital cardiac arrest, OHCA out-ofhospital cardiac arrest, TTM targeted temperature management

values during the first 48 hours was 3 (IQR: 0-21) and 17 (IQR: 8 - 49) minutes in patients with a good and poor neurological outcome, respectively $(p=0.052)$. The median duration with bilateral BIS 0 values was higher in patients with a poor neurological outcome (CPC1-2: 0 (IQR: 0-3) vs. CPC3-5: 13 minutes (IQR: $0-219) ; p=0.016)$. Within the first 48 hours, the total median duration with any BIS 0 value was 8 (IQR: $1-21$ ) and 49 (IQR: 23-378) minutes in patients with a good and poor neurological outcome, respectively $(p=0.007)$. By means of a ROC analysis, a total duration of 1820 seconds (i.e. 30, 33 minutes) with BIS 0 values calculated over the first 48 hours predicted poor neurological outcome with a sensitivity of $63 \%$ (95\% CI: $41-81$ ) and specificity of $100 \%$ (95\% CI: 54-100) (AUC: 0.861 (0.719-1.000); $p=0.007$; Fig. 2). With this cut-off value, a PPV of $100 \%$ (95\% CI: 75-100), a NPV of 40\% (95\% CI: $17-67$ ) and FPR of $0 \%$ (95\% CI: 0-25) was calculated. An additional ROC analysis was performed using the total duration with BIS 0 values calculated over the first 12 hours to assess the possibility to use the BIS monitor as a triage method after OHCA. A total duration of 1810 seconds (30.17 minutes) with BIS 0 values calculated over the first 12 hours predicted poor neurological outcome with a sensitivity of 57\% (95\% CI: 34-77) and specificity of $100 \%$ (95\% CI: 54-100) (AUC: 0.855 (0.703-1.000); $p=0.008)$.

The first 48 hours after the induction of TTM at $33{ }^{\circ} \mathrm{C}$ can be subdivided in (1) a hypothermic phase $(0-24 \mathrm{~h}),(2)$ a rewarming phase (24-36 h) and (3) the normothermia phase $(36-48 \mathrm{~h})$. BIS 0 values were observed during hypothermia in all six patients with a good and in 23 out of 24 patients (96\%) with a poor neurological outcome (Fig. 3). Seven patients had BIS 0 values during the rewarming phase of whom one (16\%) with a good and five (22\%) with a poor neurological outcome. Three patients (13\%) with poor neurological outcome experienced BIS 0 values during normothermia in contrast to none of the patients 
Table 1 Demographics

\begin{tabular}{|c|c|c|c|}
\hline & Good neurological outcome $(N=6)$ & Poor neurological outcome $(N=24)$ & $P$ value \\
\hline \multicolumn{4}{|l|}{ Demographics } \\
\hline Age & $66(49-70)$ & $67(56-79)$ & 0.27 \\
\hline Male & $5(83)$ & $21(88)$ & 0.79 \\
\hline Initial rhythm & & & 0.60 \\
\hline Shockable & $4(67)$ & $17(77)$ & \\
\hline Non-shockable & $2(33)$ & $5(22)$ & \\
\hline Witnessed arrest & $6(100)$ & $20(87)$ & 0.35 \\
\hline Time to target temperature (min) & $174(90-294)$ & $147(101-229)$ & 0.85 \\
\hline Time emergency call - ROSC (min) & $38(30-38)$ & $35(22-39)$ & 0.48 \\
\hline \multicolumn{4}{|l|}{ Neuron-specific enolase $(\mu \mathrm{g} / \mathrm{l})$} \\
\hline Hour 24 & $28(21-57)$ & $86(55-110)$ & 0.006 \\
\hline Hour 48 & $48(15-62)$ & $156(71-278)$ & 0.003 \\
\hline \multicolumn{4}{|l|}{ Electro-encephalography } \\
\hline Burst suppression & $1(16)$ & $11(46)$ & 0.36 \\
\hline Status epilepticus & $0(0)$ & $10(42)$ & 0.07 \\
\hline \multicolumn{4}{|c|}{ Use of sedatives and neuromuscular blockage } \\
\hline Max. dose propofol (mg/kg/hour) & $3.30(1.55-5.78)$ & $2.20(1.78-2.53)$ & 0.14 \\
\hline Max. dose remifentanil ( $\mu \mathrm{g} / \mathrm{kg} / \mathrm{min})$ & $0.12(0.07-0.13)$ & $0.10(0.08-0.13)$ & 0.96 \\
\hline Neuromuscular blockage use & $3(50)$ & $15(63)$ & 0.66 \\
\hline
\end{tabular}

Values are shown as median with 25 and 75 percentile and $\mathrm{n}(\%)$

Significant values are indicated in bold

ROSC return of spontaneous circulation

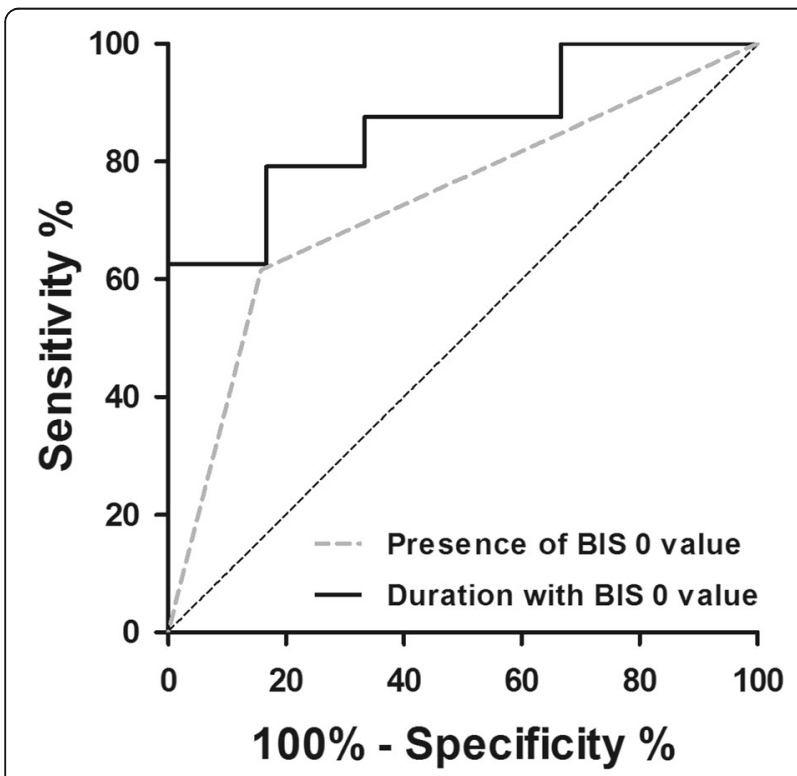

Fig. 2 ROC curves of the presence and duration with BIS 0 values. The presence of a BIS 0 value predicted poor neurological outcome with a sensitivity of $62 \%$ (95\% Cl: $45-7)$ and specificity of $84 \%$ (95\% Cl: $45-77)$ (AUC: $0.729(0.614-0.844))$. The total duration with any BIS 0 values was calculated within the first 48 hours after TTM was initiated. A duration of 1820 seconds was associated with poor neurological outcome with a sensitivity of $62 \%$ (95\% Cl: $41-81)$ and specificity of 100\% (95\% Cl: 54-100) (AUC: 0.861 (0.719-1.000)) with a good neurological outcome, corresponding to a sensitivity of $13 \%$ (95\% CI: $3-33)$ and specificity of 100\% (95\% CI: 52-100).

\section{Discussion}

This study shows that the duration with BIS 0 values rather than a single observation serves as a better outcome predictor after OHCA. Thirty OHCA patients (38\%) experienced a BIS 0 value during TTM. Still, six of them attained a good neurological outcome. In contrast, a total duration with BIS 0 values beyond half an hour was uniformly associated with poor neurological outcome at 180 days postCA. Furthermore, bilateral BIS 0 values seem to be of better prognostic value as compared to unilateral BIS 0 values.

To date, early neuroprognostication in OHCA survivors remains challenging. Current guidelines now recommend the use of a multimodal neuroprognostication algorithm containing prognostic tools such as EEG, SSEP and neuroimaging. Although these robust predictors do reach a high specificity on their own, they require trained physicians for correct interpretation and are often considered as time-consuming and expensive [4-7]. Due to its simple and non-invasive nature, BIS monitoring has been investigated for its potential to assist with early neuroprognostication. The phenomenon that OHCA patients displaying lower BIS values are 


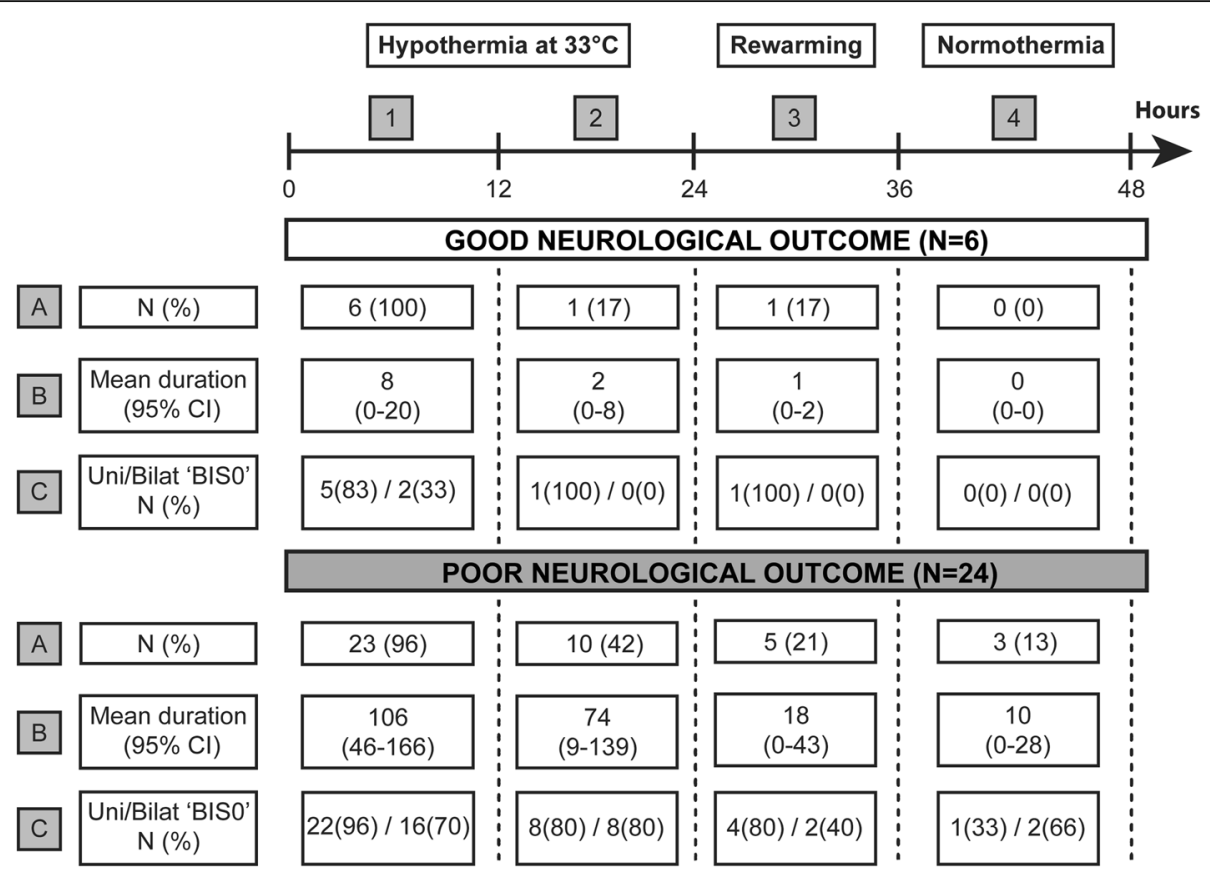

Fig. 3 Overview of the characteristics of BIS 0 values within specific time periods. In total, six patients with a good and 24 with a poor neurological outcome experienced at least one BIS 0 value within the first 48 hours following CCU admission. After subdividing this 48 -hour time period into four equal time frames (denoted as $1-4$ in the figure), the proportion of patients (a) with their respective mean duration of BIS 0 in minutes (b) was calculated per phase for both outcome groups. Additionally, the percentage of patients experiencing unilateral (BIS 0 at one hemisphere) or bilateral (BIS 0 at both hemispheres) BIS 0 values (c) is represented within each phase as well

prone to a worse neurological outcome has gained acceptance in recent years [8-14]. Several studies reported that the presence of BIS 0 values at any point up to 24 hours could be considered as an early indicator for poor outcome (specificity: 100\%) [8-10]. This was mitigated by a subsequent study in which BIS 0 values predicted poor neurological outcome with a specificity of only $90 \%$ [11]. This is consistent with our results in which the presence of a BIS 0 value corresponded with a specificity of $84 \%$. In total, six OHCA patients experienced BIS 0 values and attained a good neurological outcome at 180 days post-CA. However, this is the first study showing that the duration of periods with BIS 0 values rather than a single observation serves as a better outcome predictor after OHCA. Within the first 48 hours after TTM was initiated, all patients who experienced BIS 0 values exceeding half an hour had a poor neurological outcome (sensitivity: $63 \%$ and specificity: 100\%). Given the described confounding impact of neuromuscular activity on the BIS index, we cannot exclude the possibility that the calculated sensitivity would have been even higher if neuromuscular blockers (NMB) would have been administered continuously [9]. Interestingly, a nearly similar predictive accuracy was reached if only the first 12 hours were taken into account. This is in line with Stammet et al. which recently demonstrated that a mean BIS below 2.4 calculated over the first 6.5 hours was a certain predictor for poor outcome [14]. In this way, our findings contribute to the concept that a prolonged duration with low BIS values in the early hours after cardiac arrest could be used to guide early postcardiac arrest triage $[9,12-14,22]$.

Another remarkable observation was the lower incidence and shorter duration with bilateral BIS 0 values in the six patients with a good neurological outcome. Since we are the first to report this finding, we can only speculate that most patients with unilateral BIS 0 values might have had sufficient cerebral reserve allowing the contralateral hemisphere to recover from small time periods of cortical inactivity. Another possibility, on the other hand, is that the incidence with bilateral BIS 0 values would have been higher with the continuous administration of NMB. Hence, future studies using bilateral BIS monitoring concomitantly with continuous full EEG are required to confirm these preliminary results and need to elucidate the impact of NMB on the uni- or bilateral appearance of a BIS 0 value.

Especially during hypothermia, BIS values as low as zero can be observed in patients with good neurological outcome. It has been shown that cerebral ischemia induces an acute failure of synaptic transmission within the first minute following circulatory arrest resulting in a flat EEG [23, 24]. Therefore, our results confirmed that iso-electric EEG patterns or BIS values of 0 are not 
uncommon in the early hours after cardiac arrest, but do not preclude full recovery of brain function. On the other hand, $25 \%$ of patients with poor neurological outcome experienced BIS 0 values after the hypothermic phase was ended. This is consistent with published data where the presence of an initial flat EEG during TTM at $33{ }^{\circ} \mathrm{C}$ was shown to be of no prognostic value and more importantly, an EEG pattern evolving from flat towards continuous EEG lines was predictive for good neurological outcome [25-30]. In analogy with these results, others demonstrated that the presence of iso-electric or low-voltage EEGs 24 hours after resuscitation, but not earlier, was a strong indicator of poor neurological outcome (specificity: 100\%) [6, 31-33]. As such, our results strengthen the hypothesis that persisting suppression of cortical activity after the end of TTM at $33{ }^{\circ} \mathrm{C}$ is associated with an increased mortality risk. Nevertheless, one should be aware that BIS indices remain an automated calculation of frontal EEG activity and, unlike conventional EEG, are not reliable to use for diagnostic purposes [30, 34].

This study has several limitations. First, BIS values were not blinded because signal quality assessments required visual confirmation. Despite this, BIS 0 values were never used in the decision to withdraw life support. In fact, treating physicians were cardiologists who were most often not familiar with the use of BIS monitoring. Second, previous studies showed that hypothermia lowers the BIS value $[35,36]$. However, it was unlikely TTM affected our results as the applied temperature regimen was uniform in all patients and the time to target temperature was not different between both outcome groups. Still, the potency of the duration with BIS 0 values to predict poor neurological outcome in patients treated with TTM at $36^{\circ} \mathrm{C}$ remains unanswered, as TTM at $33{ }^{\circ} \mathrm{C}$ was applied in our patient cohort. Third, the exact time to return of spontaneous circulation (ROSC) and the specific time span between ROSC and the initiation of BIS monitoring were unknown, which could be considered as a limitation. In order to have a similar starting point for all patients, it was therefore decided to synchronize the raw sampled BIS data with the time TTM at $33{ }^{\circ} \mathrm{C}$ was initiated. Fourth, the total sample size was relatively small although $39 \%$ of all patients experienced BIS 0 values. Due to the limited sample size, it is not possible to exclude that in a larger database, OHCA patients presenting with BIS 0 values exceeding half an hour, might attain a good neurological outcome. Therefore, this (preliminary) study should be considered as a hypothesis-generating one which raised some interesting thoughts that should be confirmed in future large-scale trials. In addition, these studies should focus on the contribution of the duration with BIS 0 values to the standardized neuroprognostication algorithm recommended by current guidelines.

\section{Conclusions}

This study demonstrated at first that a prolonged duration with BIS 0 values serves as a better outcome predictor after OHCA as compared to a single observation. Although less specific, isolated BIS 0 values remained associated with a poor neurological outcome. Despite these promising results, the validity of this prognostic parameter in clinical practice remains inconclusive due to the small number of patients studied.

\section{Abbreviations}

BIS: Bispectral index; BIS 0: BIS values equal to zero; CA: Cardiac arrest; CCU: Coronary care unit; CPC: Cerebral performance category; EEG: Electroencephalography; EMG: Electromyographic power; FPR: False-positive range; IQR: Interquartile range; NMB: Neuromuscular blocker; NPV: Negative predictive value; OHCA: Out-of-hospital cardiac arrest; PPV: Positive predictive value; ROC: Receiver operating characteristic; ROSC: Return of spontaneous circulation; SQI: Signal quality index; SSEP: Somatosensory evoked potential; TTM: Targeted temperature management

\section{Acknowledgements}

The authors wish to thank the residents, nursing and medical staff of the coronary care unit of Ziekenhuis Oost-Limburg for their cooperation and support in this study.

\section{Funding}

This work was supported by the Limburg Clinical Research Program (LCRP) UHasselt-ZOL-Jessa, supported by the foundation Limburg Sterk Merk, Hasselt University, Ziekenhuis Oost-Limburg and Jessa Hospital.

\section{Availability of data and materials}

The datasets used and/or analysed during the current study are available from the corresponding author on reasonable request.

\section{Authors' contributions}

WE was responsible for the study execution, data management, data analysis, data interpretation, and manuscript writing. CG was responsible for the study design, study execution, oversight of data management, data interpretation and critically revised the manuscript. GH was responsible for data analysis and critically revised the manuscript. WB and FJ were responsible for study design, interpretation of results and manuscript editing. JD and CDD were responsible for the conception, study design, study execution, data interpretation, and manuscript editing. All the authors read and approved the final manuscript.

Ethics approval and consent to participate

Ethical approval was obtained before study onset from the local medical ethics committee (Comité Medische Ethiek Ziekenhuis Oost-Limburg 11/066). Written informed consent was obtained from the patient's next of kin.

Consent for publication

Not applicable.

Competing interests

The authors declare that they have no competing interests.

\section{Publisher's Note}

Springer Nature remains neutral with regard to jurisdictional claims in published maps and institutional affiliations.

\section{Author details}

'Department of Medicine and Life Sciences, Hasselt University, Diepenbeek, Belgium. ${ }^{2}$ Department of Anaesthesiology, Intensive Care, Emergency Medicine and Pain Therapy, Ziekenhuis Oost-Limburg, Genk, Belgium. ${ }^{3}$ Department of Cardiology, Ziekenhuis Oost-Limburg, Genk, Belgium. 
Received: 25 April 2017 Accepted: 1 August 2017

Published online: 22 August 2017

\section{References}

1. Lemiale V, Dumas F, Mongardon N, Giovanetti O, Charpentier J, Chiche JD, et al. Intensive care unit mortality after cardiac arrest: the relative contribution of shock and brain injury in a large cohort. Intensive Care Med. 2013;39(11):1972-80.

2. Dragancea I, Rundgren M, Englund E, Friberg H, Cronberg T. The influence of induced hypothermia and delayed prognostication on the mode of death after cardiac arrest. Resuscitation. 2013;84(3):337-42.

3. Nielsen N, Wetterslev J, Cronberg T, Erlinge D, Gasche Y, Hassager C, et al. Targeted temperature management at 33 degrees $C$ versus 36 degrees $C$ after cardiac arrest. N Engl J Med. 2013;369(23):2197-206.

4. Nolan JP, Soar J, Cariou A, Cronberg T, Moulaert VR, Deakin CD, et al. European Resuscitation Council and European Society of Intensive Care Medicine Guidelines for Post-resuscitation Care 2015: Section 5 of the European Resuscitation Council Guidelines for Resuscitation 2015. Resuscitation. 2015;95:202-22.

5. Bouwes A, Binnekade JM, Kuiper MA, Bosch FH, Zandstra DF, Toornvliet AC, et al. Prognosis of coma after therapeutic hypothermia: a prospective cohort study. Ann Neurol. 2012;71(2):206-12

6. Hofmeijer J, Beernink TM, Bosch FH, Beishuizen A, Tjepkema-Cloostermans MC, van Putten MJ. Early EEG contributes to multimodal outcome prediction of postanoxic coma. Neurology. 2015;85(2):137-43.

7. Hirsch KG, Mlynash M, Jansen S, Persoon S, Eyngorn I, Krasnokutsky MV, et al. Prognostic value of a qualitative brain MRI scoring system after cardiac arrest. J Neuroimaging. 2015;25(3):430-7.

8. Stammet $P$, Werer $C$, Mertens L, Lorang C, Hemmer M. Bispectral index (BIS) helps predicting bad neurological outcome in comatose survivors after cardiac arrest and induced therapeutic hypothermia. Resuscitation. 2009; 80(4):437-42.

9. Seder DB, Fraser GL, Robbins T, Libby L, Riker RR. The bispectral index and suppression ratio are very early predictors of neurological outcome during therapeutic hypothermia after cardiac arrest. Intensive Care Med. 2010;36(2):281-8.

10. Leary M, Fried DA, Gaieski DF, Merchant RM, Fuchs BD, Kolansky DM, et al. Neurologic prognostication and bispectral index monitoring after resuscitation from cardiac arrest. Resuscitation. 2010;81(9):1133-7.

11. Stammet $P$, Wagner DR, Gilson G, Devaux $Y$. Modeling serum level of s100beta and bispectral index to predict outcome after cardiac arrest. J Am Coll Cardiol. 2013;62(9):851-8.

12. Seder DB, Dziodzio J, Smith KA, Hickey P, Bolduc B, Stone P, et al. Feasibility of bispectral index monitoring to guide early post-resuscitation cardiac arrest triage. Resuscitation. 2014;85(8):1030-6.

13. Selig C, Riegger C, Dirks B, Pawlik M, Seyfried T, Klingler W. Bispectral index (BIS) and suppression ratio (SR) as an early predictor of unfavourable neurological outcome after cardiac arrest. Resuscitation. 2014;85(2):221-6.

14. Stammet $P$, Collignon O, Werer C, Sertznig C, Devaux Y. Bispectral index to predict neurological outcome early after cardiac arrest. Resuscitation. 2014; 85(12):1674-80.

15. Burjek NE, Wagner CE, Hollenbeck RD, Wang L, Yu C, McPherson JA, et al. Early bispectral index and sedation requirements during therapeutic hypothermia predict neurologic recovery following cardiac arrest. Crit Care Med. 2014:42(5):1204-12

16. Sandroni C, Cariou A, Cavallaro F, Cronberg T, Friberg H, Hoedemaekers C, et al. Prognostication in comatose survivors of cardiac arrest: an advisory statement from the European Resuscitation Council and the European Society of Intensive Care Medicine. Resuscitation. 2014;85(12):1779-89.

17. Meex I, Dens J, Jans F, Boer W, Vanhengel K, Vundelinckx G, et al. Cerebral tissue oxygen saturation during therapeutic hypothermia in post-cardiac arrest patients. Resuscitation. 2013;84(6):788-93.

18. Genbrugge C, Eertmans W, Meex I, Van Kerrebroeck M, Daems N, Creemers $\mathrm{A}$, et al. What is the value of regional cerebral saturation in post-cardiac arrest patients? A prospective observational study. Crit Care. 2016;20(1):327.

19. Murray MJ, DeBlock H, Erstad B, Gray A, Jacobi J, Jordan C, et al. Clinical practice guidelines for sustained neuromuscular blockade in the adult critically ill patient. Crit Care Med. 2016;44(11):2079-103.

20. Wijdicks EF, Hijdra A, Young GB, Bassetti CL, Wiebe S, Quality Standards Subcommittee of the American Academy of Neurology. Practice parameter: prediction of outcome in comatose survivors after cardiopulmonary resuscitation (an evidence-based review): report of the Quality Standards
Subcommittee of the American Academy of Neurology. Neurology. 2006; 67(2):203-10.

21. Grenvik A, Safar P. Brain failure and resuscitation. New York: Churchill Livingstone; 1981. p. 268.

22. Riker RR, Stone Jr PC, May T, McCrum B, Fraser GL, Seder D. Initial bispectral index may identify patients who will awaken during therapeutic hypothermia after cardiac arrest: a retrospective pilot study. Resuscitation. 2013:84(6):794-7.

23. Hofmeijer J, van Putten MJ. Ischemic cerebral damage: an appraisal of synaptic failure. Stroke. 2012;43(2):607-15.

24. van Dijk JG, Thijs RD, van Zwet E, Tannemaat MR, van Niekerk J, Benditt DG, et al. The semiology of tilt-induced reflex syncope in relation to electroencephalographic changes. Brain. 2014;137(Pt 2):576-85.

25. Rundgren M, Rosen I, Friberg H. Amplitude-integrated EEG (aEEG) predicts outcome after cardiac arrest and induced hypothermia. Intensive Care Med. 2006;32(6):836-42

26. Rundgren M, Westhall E, Cronberg T, Rosen I, Friberg H. Continuous amplitude-integrated electroencephalogram predicts outcome in hypothermia-treated cardiac arrest patients. Crit Care Med. 2010;38(9):1838-44.

27. Jorgensen EO, Holm S. The natural course of neurological recovery following cardiopulmonary resuscitation. Resuscitation. 1998;36(2):111-22.

28. Crepeau AZ, Rabinstein AA, Fugate JE, Mandrekar J, Wijdicks EF, White RD, et al. Continuous EEG in therapeutic hypothermia after cardiac arrest: prognostic and clinical value. Neurology. 2013:80(4):339-44.

29. Tjepkema-Cloostermans MC, Hofmeijer J, Beishuizen A, Hom HW, Blans MJ, Bosch FH, et al. Cerebral Recovery Index: reliable help for prediction of neurologic outcome after cardiac arrest. Crit Care Med. 2017;45(8):e789-97.

30. Hofmeijer J, van Putten MJ. EEG in postanoxic coma: Prognostic and diagnostic value. Clin Neurophysiol. 2016;127(4):2047-55.

31. Cloostermans MC, van Meulen FB, Eertman CJ, Hom HW, van Putten MJ. Continuous electroencephalography monitoring for early prediction of neurological outcome in postanoxic patients after cardiac arrest: a prospective cohort study. Crit Care Med. 2012;40(10):2867-75.

32. Tjepkema-Cloostermans MC, Hofmeijer J, Trof RJ, Blans MJ, Beishuizen A, van Putten MJ. Electroencephalogram predicts outcome in patients with postanoxic coma during mild therapeutic hypothermia. Crit Care Med. 2015; 43(1):159-67.

33. Sivaraju A, Gilmore EJ, Wira CR, Stevens A, Rampal N, Moeller JJ, et al. Prognostication of post-cardiac arrest coma: early clinical and electroencephalographic predictors of outcome. Intensive Care Med. 2015; 41(7):1264-72

34. Westhall $E$, Rossetti AO, van Rootselaar AF, Wesenberg Kjaer T, Horn J, Ullen $S$, et al. Standardized EEG interpretation accurately predicts prognosis after cardiac arrest. Neurology. 2016;86(16):1482-90.

35. Mathew JP, Weatherwax KJ, East CJ, White WD, Reves JG. Bispectral analysis during cardiopulmonary bypass: the effect of hypothermia on the hypnotic state. J Clin Anesth. 2001;13(4):301-5.

36. Honan D, Doherty D, Frizelle H. A comparison of the effects on bispectral index of mild vs. moderate hypothermia during cardiopulmonary bypass. Eur J Anaesthesiol. 2006;23(5):385-90.

\section{Submit your next manuscript to BioMed Central and we will help you at every step:}

- We accept pre-submission inquiries

- Our selector tool helps you to find the most relevant journal

- We provide round the clock customer support

- Convenient online submission

- Thorough peer review

- Inclusion in PubMed and all major indexing services

- Maximum visibility for your research

Submit your manuscript at www.biomedcentral.com/submit 